\title{
Neoliberalismo y procesos de privatización "en" la educación pública en Inglaterra y España
}

\author{
Cristina Pulido-Montes \\ Universidad de Valencia \\ Luis Miguel Lázaro Lorente \\ Universidad de Valencia
}

\section{Resumen}

El neoliberalismo desde la década de los 80 ha impactado en las agendas de política educativa tanto nacionales como internacionales con el objetivo de introducir la racionalidad competitiva en el seno de los sistemas educativos. En gran medida, estas políticas introducen mecanismos que ponen a funcionar a los centros educativos bajo formas pedagógicas, de gestión y de gobierno similares a las de una empresa. No en vano, el impacto y profundidad de estas reformas están relacionadas con la historia, contexto, economía y objetivos de los responsables políticos en el poder en un momento dado. Por lo tanto, no podemos hablar de un neoliberalismo, sino de diversos neoliberalismos. En el presente artículo se desarrolla un estudio comparado e intertextual sobre el impacto de las políticas "en" privatización de la educación en Inglaterra y España, con el objetivo de estudiar la recontextualización de estas políticas según el ejecutivo en el gobierno. En el caso de Inglaterra, la privatización "en" la educación ha alcanzado los objetivos de los gobiernos conservadores y laboristas. En el caso español, la racionalidad competitiva no ha irrumpido con tanta fuerza en los objetivos políticos de la izquierda política.

Palabras clave: neoliberalismo, privatización, educación, Inglaterra, España, comparación 


\title{
Neoliberalism and Privatization Processes "In" Public Education in England and Spain
}

\begin{abstract}
Neoliberalism since the 1980s has impacted both national and international educational policy agendas with the aim of introducing competitive rationality within educational systems. To a large extent, these policies introduce mechanisms that put educational centers to work under pedagogical, management and governance forms similar to those of a company. Not surprisingly, the impact and depth of these reforms are related to the history, context, economy, and objectives of the political leaders in power at any given time. Therefore, we cannot speak of a neoliberalism, but of different neoliberalisms. This article develops a comparative and intertextual study on the impact of policies "on" privatization of education in England and Spain, with the aim of studying the recontextualization of these policies according to the executive in the government. In the case of England, privatization "in" education has achieved the goals of both the Conservative and Labor governments. In the Spanish case, competitive rationality has not broken into the political objectives of the political left with such force.
\end{abstract}

Keywords: neoliberalism, privatization, education, England, Spain, comparison

\section{Néolibéralisme et processus de privatisation « dans » l'enseignement public en Angleterre et en Espagne}

\section{Résumé}

Depuis les années 1980, le néolibéralisme a eu un impact sur les agendas politiques nationaux et internationaux en matière d'éducation dans le but d'introduire une rationalité compétitive au sein des systèmes éducatifs. Dans une large mesure, ces politiques introduisent des mécanismes qui soumettent les établissements d'enseignement à des formes pédagogiques, de gestion et de gouvernement semblables à celles d'une entreprise. II n'est pas surprenant que l'impact et la profondeur de ces réformes soient liés à l'histoire, au contexte, à l'économie et aux objectifs des dirigeants politiques au pouvoir à un moment donné. On ne peut donc pas parler d'un seul néolibéralisme, mais de divers néolibéralismes. Cet article développe une étude comparative et intertextuelle sur l'impact des politiques de privatisation "in" de l'éducation en Angleterre et en Espagne, dans le but d'étudier la recontextualisation de ces politiques en fonction de l'exécutif au gouvernement. Dans 
le cas de l'Angleterre, la privatisation « dans » l'éducation a atteint les objectifs des gouvernements conservateurs et travaillistes. Dans le cas espagnol, la rationalité concurrentielle n'a pas fait irruption dans les objectifs politiques de la gauche politique avec une telle force.

Mots-clés: néolibéralisme, privatisation, éducation, Angleterre, Espagne, comparaison

\section{Perspectiva Teórica}

La historia más reciente del neoliberalismo, como apunta Stedman Jones (2012), arranca con el coloquio organizado en 1938 en París por el filósofo Louis Rougier para debatir el futuro de la sociedad con el libro de Walter Lippmann A Critique into the Principles of the Good Society, de 1937, como tema. Años más tarde, en abril de 1947, buena parte de los asistentes a esa reunión en la capital francesa formarán la Mont Pelerin Society, en un encuentro en Vevey, Suiza, impulsada por Friedrich August von Hayek, sentando las bases de un neoliberalismo transatlántico (Stedman, 2012, p. 6). Milton Friedman, conspicuo representante de ese grupo de economistas todavía en los márgenes, se convierte pronto en un adalid de la reforma del sistema educativo acorde a los nuevos principios económicos que predica la Escuela de Chicago.

En 1955, en su trabajo seminal-The Role of Government in Education-, base de las concepciones defensoras del cheque escolar y la libre elección del centro educativo por las familias, Friedman reconoce que hay que facilitar el acceso de la ciudadanía a la educación para garantizar la estabilidad de una sociedad democrática. Sin embargo, considera que los males que aquejaban por ineficiencia al sistema público de educación eran el resultado de operar el gobierno en un régimen de monopolio cuasi "socialista" altamente burocratizado. Su solución pasaba por reducir de manera drástica el papel gubernamental en la oferta de educación dando juego a la iniciativa privada mediante escuelas "acreditadas", operadas por empresas con ánimo de lucro o instituciones que no tienen en el beneficio su principal objetivo. El rol del gobierno lo fija en garantizar unos estándares mínimos en todos los centros educativos y financiar esas oportunidades de educación por medio de cheques que las familias gastan en el tipo de educación y centro que eligen para sus hijos (Friedman, 1955).

Años más tarde, Friedman, en Selling schooling like groceries (1973), vuelve a denunciar la incapacidad del sistema de educación norteamericano que, al igual que otros servicios públicos como correos, son ineficientes porque se gestionan por agencias gubernamentales en régimen de monopolio. Justo lo contario del modelo de funcionamiento eficaz que caracteriza a las empresas privadas operando en un mercado desregulado y competitivo. Así, la ineficacia del sistema público de educación solo puede ser superada introduciendo con más intensidad los principios de competición, libre empresa y elección de centro por las familias con el correspondiente cheque escolar. En diciembre de 2005, un año antes de su muerte, los ataques organizados contra lo público, aprovechando hechos catastróficos para 
verlos como buenas oportunidades de mercado, ejemplificaban bien lo que Klein ha definido como "capitalismo del desastre" (Klein, 2007, p. 26). Friedman, en The Promise of Vouchers (2005), planteaba aprovechar la ruina de las infraestructuras del sistema público de educación en New Orleans, como consecuencia de los devastadores efectos del huracán Katrina, convirtiendo el desastre en una excelente ocasión para aprovechar la reconstrucción privatizando la oferta educativa a través del sistema de charter schools. Las escuelas públicas de la ciudad destruidas no eran buenas porque su ineficiente gestión y organización era pública. Se imponía pues aprovechar la calamidad para instalar un sistema de libre elección de centros, compitiendo entre ellos, soportado en los cheques escolares entregados a las familias. El modelo del cheque escolar ha madurado generando en Estados Unidos nuevos modelos complementarios del tradicional, como los Scholarship tax credit programs, o las Education savings accounts o las ESAs. Políticas bendecidas y legitimadas desde los think tanks neoconservadores con los que Friedman trabajó directamente como Heritage Foundation, Cato Institute, o American Enterprise Institute (Klein, 2007, p. 38), también con el mismo celo desde el Institute of Economic Affairs, el Centre for Policy Studies y el Adam Smith Institute. Todo en el marco de la "trinidad política" de la que habla Klein: "la eliminación del rol público del Estado, la absoluta libertad de movimientos de las empresas y un gasto social prácticamente nulo" (Klein, 2007, p. 38).

Ese enfoque de mercado proyectado sobre la educación no es, como sostiene Apple, la única corriente que conforma el conglomerado ideológico del neoliberalismo. Un movimiento que "toma distintas formas en diferentes contextos", y que tampoco es "un movimiento uniforme, ya que hay tendencias contradictorias dentro de él" (Apple, 2017, p. 148). Pero de una forma u otra, sea en momentos diferentes o no, son identificables los grupos que Apple establece como impulsores de la agenda educativa neoliberal, de la "modernización conservadora": los defensores de soluciones de mercado para los problemas educativos; los neoconservadores que añoran un pasado idealizado para defender un proyecto de restauración cultural asentado en un conocimiento o cultura comunes que la escuela transmite; los conservadores autoritarios populistas de carácter religioso; y los profesionales de clase media comprometidos con la "cultura de la auditoria", de la medición, de la nueva gestión, de la rendición de cuentas con la imposición externa de normas y reformas ideales derivadas de los resultados de la medición de resultados académicos de escolares, escuelas y profesorado (Apple, 2017, pp. 149-150). Les une su voluntad última de gestionar la actividad educativa con criterios de mercado, que, como sostiene Chomsky, "ha de verse como parte de un ataque general del neoliberalismo sobre lo público" (Chomsky, 2017, p. 169). Más allá de Estados Unidos, globalmente la más influyente de las tres corrientes ahora señaladas es, probablemente, la que propugna aplicar a la educación soluciones de mercado que postergan lo público en favor de lo 
privado. Autores como Ball y Youdell (2008) denominan a este tipo de formas "en"1 privatización de la educación pública, como políticas endógenas.

La proyección real del enfoque neoliberal en las políticas educativas, la construcción de la agenda neoliberal en política educativa corresponde a la que Stedman Jones denomina tercera época de esa corriente económico-política, es decir, a partir de los años 80. Para Steger y Roy (2010), esa sería la primera; la segunda en los 90 con Clinton y la defensa de la globalización de los mercados y el New Laboury la Tercera vía de Blair (Steger \& Roy, 2010, p. 21 y 50). Es, con claridad, la etapa en la que se consolida el declive de las políticas keynesianas iniciado en la década anterior, que materializan Ronald Reagan en Estados Unidos y Margaret Thatcher en el Reino Unido. La Dama de Hierro veía a Friedman como "un luchador por la libertad intelectual" (Klein, 2007, p. 42). El periodo en el que los principios esenciales del neoliberalismo se adoptan en los organismos internacionales: el Fondo Monetario Internacional (FMI), el Banco Mundial, la Organización Mundial del Comercio (OMC) y la Unión Europea (Stedman, 2012, p. 8). El momento en el que, como plantea Bourdieu (1998), se instaura un "programa de destrucción metódica de los colectivos", de todas las estructuras colectivas que pudieran obstaculizar la "lógica del mercado puro": la nación, el Estado, los sindicatos, las asociaciones, las cooperativas, los partidos, e incluso la familia; en suma, de todo colectivo orientado hacia "la consecución racional de fines colectivamente elaborados y aprobados" (Bourdieu, 1998, p. 3). La paulatina asunción de principios neoliberales se trasladará también a la izquierda socialistasocialdemócrata, con más o menos intensidad según países, con bastante claridad en el Reino Unido y también en Australia (Connell, 2013, p. 110).

Los años en los que, en el terreno de la educación y sus políticas, como sostenía Roger Standaert (1993), desde principios de racionalidad técnica y supervisión externa al sistema educativo, se despliega una panoplia de actuaciones que pasarán a ser invariantes de la actuación en política educativa tanto de los gobiernos de los países desarrollados como en desarrollo a lo largo de las siguientes tres décadas. La etapa que contempla propuestas, planteamientos y realizaciones que Hood (1991) había definido como principios esenciales de la Nueva Gestión Pública (NGP) en trasposición a la educación tales como: la introducción de las evaluaciones centrales o externas; las comprobaciones periódicas externas; el incremento de la función inspectora; la imposición centralizada de los currícula; la financiación en función de los resultados; el incremento de la presencia en los órganos de representación escolar de agentes externos; la promoción de mecanismos de mercado en la recluta del alumnado; el respaldo e influencia de los empleadores en el sistema educativo; el reforzamiento del modelo de empresa en la gestión escolar; la libertad sin restricciones de elección de centros escolares y el estudio de implantación del cheque escolar (Standaert, 1993, p. 162). Mecanismos y políticas "en" privatización de la educación que Ball y Youdell

\footnotetext{
${ }^{1}$ Las políticas "en" o endógenas en la privatización de la educación son definidas como los mecanismos, filosofía, prácticas y lógicas del mercado que se insertan en la educación pública para hacerla funcionar bajo los preceptos del mercado.
} 
(2008) categorizarían como endógenas, en la medida que ponen a funcionar a los centros educativos como verdaderas empresas.

Un concepto de educación en su base desde el que, como denuncia Nussbaum, "A ojos de muchos administradores (y padres y estudiantes), parece demasiado costoso permitirse el aparentemente inútil lujo de aprender para el enriquecimiento de la vida" (Nussbaum, 2005, p. 323). Una manera de entender el sentido último de la educación que nada tiene que ver con el objetivo de la educación que planteaba Rousseau en el Emilio, enseñar el oficio de vivir, o, en la reformulación de Morin, ayudar a aprender a vivir, a, sobre todo, fortalecer "la capacidad de afrontar los problemas fundamentales y globales del individuo, del ciudadano, del ser humano" (Morin, 2016, pp. 15-16). Una aspiración que "La tendencia tecnoeconómica, cada vez más poderosa e influyente, tiende a reducir la educación a la adquisición de competencias socioprofesionales en detrimento de las competencias existenciales" (Morin, 2016, p. 26). Es la hegemonización, cuasi global, de la "nueva escuela capitalista" que comporta un discurso de políticas educativas enteramente consagrado al concepto de "capital humano" (Laval, Vergne, Clèment y Dreux, 2012, p. 162).

Nuestro trabajo será, como fija Leonardo Morlino para la mayoría de los estudios comparados, diacrónico, ofreciendo de esta forma la potencialidad de observar los cambios (Morlino, 2018, p. 55). Se ciñe, pues, a esa tercera etapa que señalaba Stedman. Será, además, un análisis binario de comparación entre dos países (Dogan y Pelassy, 1990, p. 126; Morlino, 2018, p. 53) confiando en que, como sostiene Weller, pueda permitirnos, más allá de conocer lo que no conocíamos, saber cosas de nosotros mismos que no conocíamos (Weller, 2017, p. 934).

Analizamos aquí, por tanto, dos recorridos de política educativa diferenciados, pero que llegan a un mismo punto en lo referente a los avances de los procesos de privatización en la educación en las tres últimas décadas. Son desarrollos que muestran bien cómo las influencias del contexto y de la acción política modulan en ambos países las tendencias internacionales adaptándose, necesariamente, a los condicionantes socio-históricos de cada país. En el caso inglés generando doctrina que, desde la economía y la ideología política neoliberal, legitima esos procesos. En el caso español recibiéndolos, sobre todo en el periodo de gobierno conservador - VI y VII legislaturas- de 1996 a 2004 desde la Nueva Derecha inglesa (de Puelles, 2005), para contextualizarlos en el particular humus socioeducativo resultado de la evolución histórica de su sistema educativo, y, en parte, motivados por las influencias de los movimientos globales de reforma educativa.

El enfoque interpretativo y metodológico del que partimos se basa en lo que destacarían Bowe, Ball \& Gold (1997) sobre que las políticas son diferentes en los diversos contextos y están atravesadas por intenciones, ideas, fines, propósitos, objetivos o planes. Incluyen que la política como "texto" puede ser mapeada a través de escritos, productos, documentos, artículos, prácticas, acciones, actividades, etc. La idea principal de Bowe, Ball \& Gold (1997) es que la política es un proceso continuo, interrelacionado e influenciado de manera recíproca por diversos contextos y 
que deben tenerse en cuenta la elaboración de políticas y análisis, es decir, la política es el resultado de las fuerzas agregadas de los contextos. En este estudio se ha manejado diversa literatura y "textos" desde un enfoque "intertextual" (Fairclough, 1992) para el que se han cotejado fuentes documentales primarias (leyes, textos normativos, datos) y secundarias (artículos, notas de prensa, discursos políticos) que han enriquecido la parte descriptiva e interpretativa del trabajo para su posterior comparación en tablas de yuxtaposición que son la parte del método comparado expuesto en el artículo presente, junto a las conclusiones comparativas.

\section{La privatización "en" la educación en Inglaterra y España}

En el presente apartado, desarrollamos el análisis descriptivo de las unidades de comparación. En primer lugar, se lleva a cabo una presentación contextual de los sistemas educativos inglés y español. En segundo lugar, se presenta una tabla de yuxtaposición en la que, por etapas de gobierno en ambos contextos, muestren qué mecanismos "en" privatización de la educación han sido introducidos.

\section{Contexto educativo Inglés}

Cowen (2005) identifica dos fechas históricas en la formación del sistema educativo inglés. Por un lado, 1870, que vio la creación del sistema de educación masiva, en el que el poder del Estado constituía una mezcla de poderes de las Autoridades Locales Centrales, el sistema nacional y las Local Education Authorities (LEA). Por otro lado, de 1944, la Education Act, que supuso la ampliación del nivel de la escuela secundaria estatal, comprendiendo escuelas primarias (escuelas elitistas), escuelas secundarias modernas y escuelas técnicas.

Hasta la llegada de la Nueva Derecha al poder en la década de 1980, la principal discusión sobre educación entre las dos principales fuerzas del gobierno en la Inglaterra del siglo XX giraba en torno a la escuela integral o meritocrática. A partir de aquí, las transformaciones en el sistema educativo inglés se han traducido en el cambio paulatino de los niveles de administración en las escuelas, avanzando hacia un modelo en el que, en la actualidad, las LEA han sido relegadas a un rol subsidiario en sus funciones. Son, por tanto, los órganos de gobierno de las escuelas y los fideicomisos escolares, junto con la Secretaría de Estado de Educación, quiénes concentran la toma de decisiones. La estrategia para erosionar las LEA comenzaría a partir del ataque de la Nueva Derecha al Estado del Bienestar.

El hito de la introducción de la NGP en el sistema educativo inglés sería la Education Reform Act (ERA, 1988) (Chitty, 2009). Lejos de estar bloqueado, ha existido un consenso generalizado en estas reformas entre conservadores y laboristas, tras el giro de estos últimos en la década de 1990 hacia la Tercera Vía. El Nuevo Laborismo de 
Tony Blair concibió el sector económico y empresarial como estratégico para la mejora de la calidad educativa y los resultados escolares.

Fue a partir del 2000 cuando el Laborismo comenzó a introducir una fusión de tipos de escuelas autónomas financiadas con fondos públicos en Inglaterra llamadas academy schools. Estos cambios precedieron a la llegada al poder del gobierno conservador-liberal demócrata liderado por David Cameron y Nick Clegg (2010-2015). A partir de ese momento, se inició la privatización masiva del sistema educativo inglés con la introducción de nuevos tipos de escuelas autónomas financiadas con fondos públicos (Converter Academies y Free Schools). El objetivo del gobierno era transformar todo el sistema educativo inglés en escuelas de tipo academy. La rendición de cuentas y la inspección relacionada con los resultados mejoraron la transición de las maintained schools (gestionadas y financiadas con fondos públicos) hacia las academies, respaldadas por informes, resultados y toma de decisiones externas. Los gobiernos conservadores posteriores de David Cameron (2015-2016), Theresa May (2016-2018) y el gobierno actual de Boris Johnson continúan las políticas de transición de maintained schools a academy schools.

\section{Contexto educativo Español}

Tras cuarenta años de dictadura en España, en 1978, se aprobó la Constitución española (CE), y con ella, el artículo 27 relativo al derecho a la educación y las libertades de enseñanza. El estudio de la trayectoria del artículo 27 revela la división de dos Españas que comprendieron la importancia de la educación para una España democrática. La resolución del citado artículo estuvo lejos de ser conflictiva, más bien articuló, en un solo nivel (artículo 27.1), el derecho a la educación y las libertades de enseñanza (Tiana, 2018). España, bajo la dictadura franquista, construyó la escuela pública nacional-católica sobre la base de esa fe y las subvencionó indiscriminadamente. Esto ha dado lugar a la actual red de escuelas privadasconcertadas que, junto con las escuelas públicas, crean la estructura de las instituciones escolares en el sistema educativo español. Las escuelas concertadas españolas son un tipo de asociación público-privada (APP), parte de la estrategia del PSOE en el año 1985 para tratar de homologarlas a las escuelas públicas.

Paralelamente, la CE propició el desarrollo del sistema de Comunidades Autónomas (CCAA), con competencias propias. El traspaso del poder educativo a las 17 CCAA se realizó por fases y alcanzó su culminación en el año 2000.

La descentralización de las regiones y competencias educativas ha derivado en un sistema de sistemas educativos (Bonal, Rambla, Calderón, y Pros, 2005) en el que el signo político de cada CCAA ha determinado el equilibrio hacia el desarrollo de políticas propias de la NGP como son los casos de Cataluña (Verger, Prieto, Pagès, y Villamor, 2018) y Andalucía (Molina-Pérez y Luengo, 2020), o la introducción de 
reformas privatizadoras endógenas y exógenas que promuevan la creación de un cuasimercado educativo, como en el caso de Madrid (Prieto y Villamor, 2013).

La ley orgánica reciente que regularía el sistema educativo español fue la Ley Orgánica para la Mejora de la Calidad Educativa (LOMCE) de 2013, promulgada por el Partido Popular (PP). En la actualidad una coalición de gobierno formada por fuerzas del espectro ideológico de la izquierda política española ha aprobado la Ley Orgánica 3/2020, de 29 de diciembre, por la que se modifica la Ley Orgánica 2/2006, de 3 de mayo, de Educación (LOMLOE), derogando la LOMCE e introduciendo importantes modificaciones sobre la LOE (2006).

\section{Políticas "en" privatización de la educación en Inglaterra y España}

En la siguiente tabla se exponen las políticas "en" privatización de la educación en Inglaterra y España de manera comparada. Las políticas "en" privatización de la educación vienen definidas por la introducción de mecanismos propios de la cultura, y prácticas de la empresa para hacer funcionar a los centros educativos bajo mecanismos similares. En el corazón de estas reformas se encuentra la NGP y los elementos que permiten auditar los centros escolares de manera externa e interna. Tras el cotejo de las diferentes fuentes documentales y de los textos normativos, jurídicos, manifiestos electorales, informes y literatura académica relacionada con la temática abordada, se presentan en la siguiente tabla de yuxtaposición los mecanismos "en" privatización de la educación en Inglaterra y España organizados por etapas de gobierno, siguiendo como criterio las políticas descritas en el marco teórico por Standaert (1993).

\section{Tabla 1}

Comparación de los mecanismos "en" privatización de la educación en Inglaterra y España según el ejecutivo en el poder

\begin{tabular}{llll}
\hline Ejecutivo & Inglaterra & Ejecutivo & España \\
\hline Partido Conservador de & Open enrollment (1981) & Partido Socialista & Conciertos educativos \\
Margareth Thatcher & Pago por mérito & Obrero Español de & (1985) \\
(1979-1991) & docente (1987) & Felipe González & Introducción \\
& Local Management & $(1982-1996)$ & generancialismo \\
& Schools (1988) & & directivo (1995) \\
& Recentralización & & Introducción en el \\
& currículum por etapas & & Consejo Escolar de un \\
& (1988) & representante del \\
Representación & & sector empresarial \\
& empleadores privados & & $(1995)$ \\
& en órganos de gobierno & & \\
& (1988) & \\
& Creación tipos de & & \\
& escuelas con mayor & & \\
autonomía (1988) & & \\
\hline
\end{tabular}




\begin{tabular}{|c|c|c|c|}
\hline Ejecutivo & Inglaterra & Ejecutivo & España \\
\hline & $\begin{array}{l}\text { Evaluación alumnado } \\
\text { por etapas (1988) } \\
\text { Financiación por } \\
\text { alumno (1988) }\end{array}$ & & \\
\hline $\begin{array}{l}\text { Partido Conservador de } \\
\text { John Major (1991-1997) }\end{array}$ & $\begin{array}{l}\text { Ránquines públicos } \\
\text { resultados evaluaciones } \\
\text { (1992) } \\
\text { Creación de la } \\
\text { inspección privada } \\
\text { (Ofsted) (1992) } \\
\text { Especialización } \\
\text { curricular de escuelas } \\
\text { (1992) } \\
\text { Incentivos para que las } \\
\text { escuelas se conviertan } \\
\text { en autónomas (1993) }\end{array}$ & $\begin{array}{l}\text { Partido Popular de } \\
\text { José Ma Aznar 1996- } \\
\text { 2004) }\end{array}$ & $\begin{array}{l}\text { Introducción Planes de } \\
\text { Mejora basados en } \\
\text { modelos de gestión y } \\
\text { evaluación } \\
\text { empresariales (1998) } \\
\text { Los órganos de } \\
\text { participación son } \\
\text { relegados a una } \\
\text { función consultiva } \\
\text { (2002) } \\
\text { El equipo directivo } \\
\text { concentra mayor poder } \\
\text { de decisión y la } \\
\text { Administración } \\
\text { acredita la dirección } \\
\text { (2002) } \\
\text { Aumento de las } \\
\text { posibilidades de } \\
\text { libertad de elección de } \\
\text { centro (1997) y de } \\
\text { dotación de conciertos } \\
\text { educativos (2002) }\end{array}$ \\
\hline $\begin{array}{l}\text { Partido Laborista de } \\
\text { Tony Blair (1997-2007) }\end{array}$ & $\begin{array}{l}\text { Introducción de } \\
\text { programas para la } \\
\text { mejora de escuelas de } \\
\text { bajos resultados (1998) } \\
\text { Nuevos tipos de } \\
\text { escuelas con mayor } \\
\text { autonomía (1998-2000) } \\
\text { Contratación directores } \\
\text { de excelencia para } \\
\text { mejorar bajos } \\
\text { resultados de escuelas } \\
(1999)\end{array}$ & $\begin{array}{l}\text { Partido Socialista } \\
\text { Obrero Español de } \\
\text { José Luís Rodríguez } \\
\text { Zapatero (2004- } \\
\text { 2011) }\end{array}$ & $\begin{array}{l}\text { Evaluaciones de } \\
\text { diagnóstico (2006) } \\
\text { Educación concertada } \\
\text { y pública como } \\
\text { servicio público (2006) }\end{array}$ \\
\hline $\begin{array}{l}\text { Partido Laborista de } \\
\text { Gordon Brown (1997- } \\
\text { 2010) }\end{array}$ & $\begin{array}{l}\text { Continuidades políticas } \\
\text { anteriores }\end{array}$ & $\begin{array}{l}\text { Partido Popular de } \\
\text { Mariano Rajoy (2011- } \\
\text { 2018) }\end{array}$ & $\begin{array}{l}\text { Pruebas reválidas de } \\
\text { evaluación del } \\
\text { alumnado } 4^{\circ} \text { ESO y } \\
\text { Bachillerato (2013) } \\
\text { Publicación de } \\
\text { resultados (2013) } \\
\text { Evaluación externa } \\
\text { alumnado educación } \\
\text { primaria ( } 3^{\circ} \text { y } 6^{\circ} \text { curso) } \\
\text { Especialización } \\
\text { curricular de centros } \\
\text { educativos (2013) } \\
\text { Itinerarios educativos } \\
\text { tempranos (2013) } \\
\text { Mayor poder de toma } \\
\text { de decisiones en el } \\
\text { director (2013) } \\
\text { Figura representante } \\
\text { municipal de los } \\
\text { Consejos Escolares } \\
\text { excluida (2013) } \\
\text { Consejo Escolar con } \\
\text { un rol consultivo (2013) } \\
\text { Fomento de la red de } \\
\text { conciertos (ampliación }\end{array}$ \\
\hline
\end{tabular}




\begin{tabular}{|c|c|c|c|}
\hline Ejecutivo & Inglaterra & Ejecutivo & España \\
\hline & & & $\begin{array}{l}\text { contratos, escuelas } \\
\text { segregadas, concesión } \\
\text { vía demanda } \\
\text { justificada, etc.) (2013) }\end{array}$ \\
\hline $\begin{array}{l}\text { Partido Conservador de } \\
\text { David Cameron y } \\
\text { Partido liberal- } \\
\text { demócrata de Nick } \\
\text { Clegg (2010-2015) } \\
\text { Partido Conservador } \\
\text { David Cameron (2015- } \\
2016)\end{array}$ & $\begin{array}{l}\text { Creación nuevos tipos } \\
\text { de escuelas privadas } \\
\text { financiadas con fondos } \\
\text { públicos (2010-2011) } \\
\text { Creación de los } \\
\text { Regional } \\
\text { Commissioners Schools } \\
\text { (2014) } \\
\text { Escuelas de bajos } \\
\text { resultados debían } \\
\text { convertirse a escuelas } \\
\text { privadas financiadas } \\
\text { con fondos públicos } \\
\text { (2016) }\end{array}$ & $\begin{array}{l}\text { Partido Socialista } \\
\text { Obrero Español de } \\
\text { Pedro Sánchez } \\
(2018-2020)\end{array}$ & $\begin{array}{l}\text { Paralización de las } \\
\text { reformas LOMCE } \\
(2013)\end{array}$ \\
\hline $\begin{array}{l}\text { Partido Conservador de } \\
\text { Theresa May (2016- } \\
\text { 2018) }\end{array}$ & $\begin{array}{l}\text { Continuar con las } \\
\text { políticas de crecimiento } \\
\text { de las escuelas privadas } \\
\text { con fondos públicos }\end{array}$ & $\begin{array}{l}\text { Partido Socialista } \\
\text { Obrero Español de } \\
\text { Pedro Sánchez } \\
\text { (2020-act.) }\end{array}$ & $\begin{array}{l}\text { Paralización de las } \\
\text { reformas LOMCE } \\
\text { (2013) } \\
\text { Evaluaciones } \\
\text { diagnósticas (2020) }\end{array}$ \\
\hline $\begin{array}{l}\text { Partido Conservador de } \\
\text { Boris Johnson (2018- } \\
\text { act.) }\end{array}$ & $\begin{array}{l}\text { Continuar con las } \\
\text { políticas de crecimiento } \\
\text { de las escuelas privadas } \\
\text { con fondos públicos }\end{array}$ & & \\
\hline
\end{tabular}

Fuente: Elaboración propia.

En el año 1979, Margareth Thatcher, una confesa neoliberal que contaba con una estrecha relación con Friedrich Von Hayeck y que habría fundado junto a dos colegas el Center for Policies Studies, llegaría a la presidencia de gobierno del Reino Unido. La Dama de Hierro, significaría una revolución y un cambio en la hoja de ruta del Partido Conservador, ya que mantuvo en parte el conservadurismo, pero acomodaría en sus líneas políticas y discursivas el enfoque neoliberal, incluso llegando a contar con una mayor presencia en las líneas estratégicas del partido. A esta puesta en práctica de lo que se conceptualizaría como neoconservadurismo, se le denominaría Nueva Derecha. Resulta imprescindible describir estas cuestiones, si atendemos a que las políticas y mecanismos introducidos en los años de gobierno de Thatcher han resignificado las políticas adoptadas por la principal oposición, el Partido Laborista y las del suyo propio hasta la actualidad. Estos argumentos son fácilmente identificables en la esfera de la educación si atendemos a que el Partido Conservador, entre 1979 y 1991. introdujo las bases del cuasimercado educativo inglés (Fitz y Gorard, 2002). Parte de estas reformas se materializarían mediante políticas tales como: el sistema de matrícula abierto (open enrollment) para resignificar el rol de las familias como consumidores electores; la introducción de la política performativa del pago por mérito docente; la recentralización del currículum en el Estado; la introducción de pruebas de evaluación externas como sistema de control y rendición de cuentas; la introducción de un modelo de gestión en el que las escuelas eran las encargadas de sus propios 
presupuestos (/ocal management schoo/s); la inclusión de figuras externas empresariales en los órganos de gobierno; la creación de nuevos tipos de escuelas autónomas de las LEA (City Technology Colleges y Graint-maintained schools) y la generalización del sistema de financiación por alumno matriculado. La regulación legal que supuso la introducción de la mayoría de estos mecanismos está en la Education Reform Act (1988). Disposición ampliamente analizada como una política propiamente thatcherista y particularista, incluso criticada por el sector neoliberal, ya que se dieron procesos de nacionalización, aunque con una clara voluntad de privatización. Como, por ejemplo, adaptando el currículum a las necesidades del mercado y ejerciendo un mayor control sobre el mismo (Lawton, 1994).

En el año 1991, John Major asume su cargo de primer ministro conservador y desarrolla una línea de continuidad con las políticas del gobierno predecesor. Aunque daría un paso más allá fortaleciendo las políticas de rendición de cuentas y del control estatal externo sobre los resultados publicándolos para fortalecer el rol de las familias como consumidoras. Además, crearía la figura de la inspección privada, la Office for Standards in Education (Ofsted), como parte de la estrategia del denominado "gobierno en la distancia" (Ball, 1993), es decir, dotar de libertad a los centros educativos y autonomía, pero monitorizada de manera externa mediante la rendición de cuentas y el control. En los años del ejecutivo Major, se introduciría una política que reforzaría la competitividad y la carrera de las escuelas por atraer al alumnado del que dependía su financiación otorgando la posibilidad a los centros educativos de especializarse en alguna temática curricular concreta. En la línea de desgaste del poder de las LEA, se dotaría de incentivos a los centros educativos que transitaran hacia modelos de escuelas más autónomas en relación con su gestión y toma de decisiones.

En el año 1997 ganaría las elecciones un joven Tony Blair que significó para el laborismo lo que Thatcher para su partido, es decir, viró la política del Partido Laborista de bases socialdemócratas y laboristas hacia políticas de tercera vía (Guiddens, 1999). Esta resignificación del partido supuso la cooperación estratégica entre el Estado y el mercado justificado por el statu quo del momento marcado por la necesidad de desarrollar políticas en una sociedad global cambiante en la que se había asumido que el neoliberalismo había llegado para quedarse y la socialdemocracia debía modernizarse. De este modo, el modelo de Estado sobre el que se construirían las políticas del Nuevo Laborismo sería el del Estado competitivo (Cerny, 1992), lo que supondría el fin del sistema nacional localmente administrado y la afirmación de la economía del conocimiento y de nuevas formas de trabajo (Ball, 2007). Es en esta etapa en la que se introducen programas de mejora sobre las "escuelas que fallan" (bajos estándares) vía los resultados de las pruebas de evaluación externas introducidas por las administraciones neoconservadoras y los informes de la Ofsted. La creencia de que la gestión y la financiación privadas podrían beneficiar a los servicios públicos en términos de mejora de la calidad hizo que las administraciones laboristas de Blair y de Gordon Brown (1997-2010) no cuestionasen 
las citadas pruebas de evaluación, la publicación de resultados, y el creciente e influyente rol de la Ofsted en el funcionamiento de las escuelas en un régimen postpanóptico (Courtney, 2016; Perryman, Maguire, Braun \& Ball, 2018).

Es en esta etapa de gobierno en la que se desplegarían una serie de reformas como el programa Fresh Start (1999), que permitió a los directivos de los centros exitosos reubicarse en centros de bajo rendimiento e implementar sus modelos de gestión a cambio de remuneraciones ad hoc. Estas políticas fueron seguidas por otras basadas en evidencias y resultados en la búsqueda de estándares mejorados, como Education Action Zones (1998), Excellence in Cities (1999), Sponsor academies (2000) y la creación de trust schools (2006). Los citados programas y tipos de escuelas se enfocarían en la mejora de los resultados académicos de los estudiantes mediante estrategias de asesoría desde escuelas de alto rendimiento hacia escuelas de bajo rendimiento y la dotación de una mayor autonomía respecto de las LEA en la toma de decisiones pedagógicas.

Ahora bien, si en algo se han puesto de acuerdo los académicos que han estudiado la privatización de la educación en Inglaterra en la última década, es en señalar que a partir del año 2010 es donde se debe situar la fecha y la alerta de la puesta en jaque mate del modelo de educación estatal inglés (Hilton, 2019). Cuando las políticas desarrolladas por el gobierno de coalición conservador y liberaldemócrata (2010-2015) fomentarían nuevas formas de escuelas apoyadas en un modelo híbrido de comercialización y regulación estatal para desarrollar una dinámica de mercado. Cameron apoyó su modelo de Estado en la filosofía de la Big Society del conservadurismo compasivo, tratando de superar el thatcherismo que había marcado profundamente el sentido del Partido Conservador teniendo por objetivo la creación de un clima que empoderase a las personas para restar poder a los políticos (Conservative Party, 2010). La traducción de la citada filosofía en la práctica significaría una mayor presión sobre la ciudadanía y liberalización de los servicios públicos. Es importante rastrear las entrevistas de David Cameron, como la publicada en el año 2015 en el periódico The Telegraph, en la que refleja su intención de convertir todas las escuelas estatales del país en modelos de escuelas tipo academies, es decir, con un funcionamiento prácticamente similar al de las escuelas privadas (independent schools), pero sostenidas con fondos públicos. Las políticas en los gobiernos de Cameron (2010-2016) darían un paso más allá expandiendo el modelo de escuelas privadas financiadas con fondos públicos en todos los niveles (primaria y secundaria), así como la gestación de nuevos organismos de rendición de cuentas y control dirigidos a impulsar el crecimiento de este tipo de escuelas, como los Regional Commissioners Schools (2014). Empero, Cameron no alcanzó a cumplir su objetivo principal para desarrollar un modelo de escuelas basado en las free schools suecas abiertas al lucro (Conservative Party, 2010). Más allá de lo destacado, la presión para la mejora de los resultados aumentaría sobre las escuelas, las cuáles se veían beneficiadas con mayores cuotas de libertad en tanto en cuanto contaban con mejores resultados. En el año 2018 el $73 \%$ de las escuelas de educación secundaria y el 30\% 
de las escuelas de educación primaria eran de tipo academies (NAO, 2018). Además, cabe destacar que el $78 \%$ de estas escuelas operan de manera conjunta mediante cadenas o fideicomisos (DfE, 2019, p.17) y muchas de ellas funcionan con un amplio sentido corporativo y cuasi-empresarial (Male, 2017).

Las administraciones de Theresa May (2016-2018) y Boris Johnson (2018-act.) han apostado por la continuidad de estas políticas, por lo que algunos investigadores sostienen que para el año 2025 la mayoría de las escuelas en Inglaterra serán de tipo academies (Hilton 2019).

En el caso español, el contexto político previo a la CE de 1978 y, sobre todo, en el período de la dictadura franquista (1939-1975), el sistema educativo español estaría configurado sobre la base de escuelas privadas confesionales (católicas) subvencionadas y una red de escuelas públicas insuficientes con escasa inversión estatal. La historia de España ha sido la desarrollada, en lo esencial, por dos fuerzas políticas y sociales enfrentadas y dividas. Por un lado, conservadores con un proyecto de escuela confesional, católica y segregada. Por otro, progresistas con un proyecto de escuela laica e igualitaria.

Ambos proyectos de escuela históricamente enfrentados en la sociedad española quedan resueltos en un mismo nivel en el artículo 27.1 de la CE 1978 que aglutinaría en su redacción el derecho a la educación y las libertades de enseñanza. En los artículos 27.3 (libertad de los padres para elegir la educación en concepciones religiosas y morales que deseen para sus hijos), y 27.4 (libertad de todos para establecer y dirigir instituciones de enseñanza que se ajusten a las normas mínimas establecidas por el Estado) se sistematizan la libertad de creación de centros educativos y la libertad de elección de centros, lo que no ha permitido que el sistema educativo español se desarrolle desde el monopolio estatal. Así, se establecería el pluralismo educativo institucionalizado mediante un sistema dual de escuelas privadas y públicas para garantizar el derecho a la educación. Por lo que las escuelas privadas financiadas con fondos públicos (escuelas concertadas) no surgen como parte de los procesos de neoliberalización o de los movimientos globales de reforma educativa. Estas escuelas, reconocidas y desarrolladas en la LODE (1985) del PSOE, han sido definidas como fruto de políticas de privatización mediante Asociaciones PúblicoPrivadas (APPs) de corte histórico (Verger, Fontdevila y Zancajo, 2016). Otra cuestión diferente es que el PSOE trató de "publificar" estas escuelas privadas que recibían subvenciones hacia un modelo que garantizara un funcionamiento similar al de los centros públicos a cambio de su financiación (Llanos, 2019), aunque en la práctica supongan un modelo de privatización de la educación.

EI PSOE de la época de Felipe González resignificaría el partido hacia posicionamientos de centroizquierda y de convergencia con el modelo socialdemócrata europeo, renunciando a las bases ideológicas de corte marxista en el XXVIII Congreso del partido en el año 1979 (Rodríguez-Prieto, 2012). Este reposicionamiento supuso el abandono de uno de los principios más importantes de la agenda socialista, sobre la construcción de una escuela laica y única, ya que, en el 
programa electoral del PSOE de 1982, no aparece referencia alguna a este modelo de escuela. Tras la llegada al poder del PSOE en 1982, son varios los análisis que describen esta etapa como la caracterizada por la puesta en marcha de políticas propias del socioliberalismo y, no tanto, de base socialdemócrata que ocuparían más la etapa de gobierno entre 1988 y 1993 (Astudillo-Ruíz, 2004).

Más allá de estas cuestiones, durante los años de gobierno del PSOE de Felipe González (1982-1996) se haría efectiva la universalidad de la educación obligatoria, se dio un fuerte impulso a la enseñanza pública, se garantizaron los derechos de participación de la comunidad educativa en la programación general de la enseñanza y se desarrollaron leyes en materia educativa que perduran hasta la actualidad debido al consenso alcanzado para su aprobación (Tiana, 2018). No obstante, en los intentos de profesionalización de la función directiva y de la gestión de los centros, se introdujeron otras reformas con características propias de la NGP, sobre todo por lo que hace a la Ley Orgánica de Participación Evaluación y Gobierno (LOPEG, 1995). Una Ley surgida en un contexto definido por el auge de las políticas de NGP y de la crisis de la socialdemocracia. Se introducen, mediante la citada ley, sistemas de elección y nombramiento de la dirección de los centros vía la administración, y se nombra al director como el observador y agente de control del personal del centro; la evaluación de los centros educativos como medida de rendición de cuentas; la figura de un representante del sector empresarial en los Consejos Escolares y se permite la contratación externa para el desarrollo de actividades extraescolares.

Ahora bien, es en las etapas de gobierno del Partido Popular de José María Aznar (1996-2004) en las que se introducen reformas propias de la privatización en la educación como las evidenciadas previamente por Standaert (1993). El PP de Aznar habría virado hacia posiciones de centro en una estrategia por tratar de atraer a votantes conservadores liberales y de centroizquierda descontentos con el PSOE (Lavezzolo y Orriols, 2008). Inspirado en el Partido Conservador británico, el PP adoptaría una agenda propia del neoliberalismo thatcherista, que pronto se materializa en reformas educativas para la potenciación de los mecanismos de libertad de elección de centro superpuestos al derecho a la educación, la introducción de elementos performativos de control y gestión propios de la empresa (Programas de Mejora de los centros), y la rendición de cuentas. Aunque es la segunda etapa de gobierno (2000-2004) del PP en la que se acomete una reforma educativa fundamentada con claridad en principios neoconservadores, y en la que se busca introducir un mayor equilibrio hacia el crecimiento de la red de escuelas concertadas, la merma de poder de los órganos de participación y una mayor concentración de la toma de decisiones en la figura del director.

Sin embargo, tras la llegada al poder del PSOE de José Luís Rodríguez Zapatero se paralizaría la citada reforma. EI PSOE trataría de alejarse de las tesis de la tercera vía británica y adoptaría principios del republicanismo cívico de Petitt (2007). Zapatero, junto con un grupo de jóvenes progresistas, tratarían de establecer su agenda política apartada del socioliberalismo practicado por Tony Blair. Su proyecto pasaría por 
reforzar la equidad por delante de la calidad. Empero, los movimientos globales de reforma y las políticas educativas de convergencia con la Unión Europea suponen, de facto, la introducción de mecanismos propios de la privatización en la educación, como son las pruebas de evaluación externas o de diagnóstico en diferentes etapas del sistema educativo, aunque se prohibiría la publicación de sus resultados (LOE, 2006). Una de las cuestiones más criticada por diferentes sectores de la izquierda española y académica en la etapa de gobierno de Zapatero fue la realizada sobre situar las escuelas concertadas en el mismo nivel que las escuelas públicas como proveedoras del servicio público educativo (LOE, 2006, art. 108.4), considerando que se había abandonado de manera definitiva la intención de transitar hacia un sistema educativo público de máximos (Torres, 2018).

Tras la llegada al poder del PP de Mariano Rajoy se volvería a una etapa de ruptura con los principios de equidad y modernización. La LOMCE (2013), una ley aprobada vía mayoría absoluta en un contexto marcado por la crisis económica del año 2008 y las políticas de austeridad europeas, sería considerada por sus objetivos, mecanismos e impacto pretendido, una ley inspirada sobre la ERA (1988) inglesa, es decir, que supuso la introducción de una agenda basada en la NGP y la introducción de mecanismos de cuasimercado (de Puelles, 2016). Una vez más, el PP reduciría el rol del Consejo Escolar a un órgano consultivo; se introducirían pruebas de evaluación de etapa y reválidas con publicación de sus resultados; se establecieron itinerarios de elección temprana a los 13 años deteriorando el carácter compresivo del sistema educativo; y se dio un mayor impulso a los conciertos educación en su alcance y dimensión adecuándolos al principio de demanda social. El uso de esos mecanismos se justificaba vía el discurso de la cultura del esfuerzo y de la meritocracia. Valores puestos en peligro por las políticas educativas socialistas, como bien atestiguaban en PISA los mediocres resultados académicos del país, y los datos de fracaso escolar en España, deudores todos de los ejecutivos socialistas (Partido Popular, 2011). Cabe destacar, que como en el caso de la crítica desarrollada en los Black Papers en Inglaterra durante la década de los 70 sobre el rol del Center for Policies Studies para la justificación de las reformas efectuadas en las administraciones Thatcher y Major, en España, el think tank FAES asociado al PP ha venido desarrollando una línea discursiva similar sobre las políticas educativas socialistas que perseguían el mismo objetivo, es decir, diagnosticar una realidad envuelta de una pérdida de valores de la cultura del esfuerzo y de la meritocracia que habían empobrecido al sistema educativo español desde una lectura y posición conservadora (Pulido-Montes, 2020).

En la actualidad, el PSOE, tras la moción de censura practicada sobre el PP en el año 2018, paralizaría los dispositivos que pretendían desarrollar dinámicas de mercado en el sistema educativo español. 


\section{Discusión y conclusiones}

Del análisis de los mecanismos "en" privatización de la educación en ambos contextos, se puede deducir que el alcance o porosidad del neoliberalismo en las agendas educativas depende de los objetivos, ideas, planes o proyectos políticos en un momento dado (Bowe, Ball \& Gold, 1997). Es más, podemos afirmar que el neoliberalismo es una tecnología móvil, es decir, que se adapta, muta y se recontextualiza adaptando distintas formas en los distintos contextos, pero con objetivos compartidos (Ong, 2007). Como se desprende del análisis llevado a cabo en Inglaterra y España, las políticas de privatización "en" la educación se encuentran integradas en la racionalidad neoliberal, es decir, buscan o persiguen el fomento de la competitividad y el desarrollo de un cuasimercado educativo.

No obstante, en el caso de Inglaterra, paradigmático en cuánto a la puesta en marcha de las políticas neoliberales en Europa, la privatización "en" la educación ha alcanzado los objetivos de los gobiernos conservadores y laboristas. En el caso español, con una transición histórica marcada por una dictadura y el enfrentamiento entre la izquierda y la derecha, la racionalidad competitiva no ha irrumpido con tanta fuerza en los objetivos políticos de la izquierda política. Ambas afirmaciones, derivadas del análisis sobre los mecanismos "en" privatización pueden venir explicadas, en el caso inglés, por la resignificación del Partido Laborista hacia la tercera vía, en el caso español, por el rechazo del ejecutivo socialista del año 2005 al año 2011 a adoptar los principios de esta tendencia política en su agenda política. Son diversos los autores que enfatizan la continuidad de las políticas del Nuevo Laborismo con las políticas desarrolladas por los ejecutivos conservadores desde la década de los 80 . Para ellos, Blair es hijo heredero de Thatcher (Espiet-Kilty, 2015). En términos generales, desde la década de los años 80 , el impacto del neoliberalismo en el contexto inglés y la resignificación del laborismo hacia el Nuevo Laborismo, han marcado la contextualización de las políticas "en" privatización de la educación fijadas por líneas de continuidad. Sin embargo, en el caso español, podemos hablar de rupturas y no tanto de líneas de continuidad, puesto que la agenda educativa del PP a pesar de seguir las políticas educativas de inspiración neoconservadora y neoliberales británicas (de Puelles, 2005), no ha llegado a asentarse y cumplir objetivos de desarrollo un cuasimercado educativo consolidado. Ello debido a la ruptura por parte del PSOE con esas políticas que perseguiría una agenda basada en la equidad por delante de los resultados. Sin embargo, podemos decir, que las políticas propias de la NGP y de la cultura de la rendición de cuentas introducidas por el ejecutivo socialista español forman parte de las tendencias globales y los movimientos de reforma educativa, así como, con mucha claridad, de las políticas de convergencia con la Unión Europea.

Más allá de que se pueda considerar que las tendencias neoliberalizadoras en educación no han tenido un impacto similar en el sistema educativo inglés que en el español, España contaba con una red de escuelas concertadas previas a las escuelas 
privadas financiadas con fondos públicos en Inglaterra, herederas de la dictadura franquista. En este caso, la resignificación del PSOE en el año 1979 y el abandono del objetivo de una escuela única y laica, ha permitido que este sistema de escuelas conviva con las escuelas públicas hasta la actualidad y prolifere según los objetivos perseguidos por los ejecutivos en un momento dado. Sobre todo, en el marco de los gobiernos autonómicos en un país altamente descentralizado y casi con la totalidad de las competencias educativas transferidas a sus CCAA (Torres, 2014).

En términos generales, los paralelismos entre el Partido Conservador y el PP son más claros que los que se puedan establecer entre las políticas educativas desarrolladas por los ejecutivos laboristas en Inglaterra y socialistas en España. Los conservadores en ambos contextos han desarrollado políticas educativas que buscaban el control de los resultados y del currículum; la publicación de resultados; el rol de la dirección como gestor; la especialización de las escuelas; el fomento de las escuelas privadas financiadas con fondos públicos vía demanda y la componente conservadora sobre la cultura del esfuerzo por encima de la igualdad de oportunidades y equidad; lo que supone la cristalización de las tendencias descritas por Standaert (1993). De hecho, el PP ha adaptado y emulado los mecanismos y políticas del ejecutivo conservador británico (de Puelles, 2005). Sin embargo, como sostiene Viñao $(2012,2016)$, en especial para el caso español el discurso neoliberal en educación ha sido además una estrategia orientada a desmantelar o aminorar el derecho a la educación para imponer una ideología conservadora y autoritaria. Se busca, pues, con ese enfoque neoliberal "imponer una ideología y modos de pensamiento, comportamientos y organización social no liberales, tampoco totalitarios, pero sí autoritarios y conservadores, por no decir ultraconservadores" (Viñao, 2016, p. 42). Es, sin duda, una confluencia clara con algunas de las corrientes influyentes que delimita Apple (2017) para Estados Unidos.

Ambos partidos políticos han contado con think tanks que han tenido un rol importante en la generación de una red discursiva e ideológica de pensamiento y de desgaste de las políticas de la oposición (Pulido-Montes, 2020), aunque no de forma paralela. Ball y Junemann (2012) describen como una característica propia de la gobernanza en la sociedad actual la influencia que ejercen otros actores externos o privados en la política actual. En el caso de las administraciones de la izquierda de ambos países estas influencias no se evidencian de manera tan clara. En suma, hablamos de procesos neoliberales de privatización en Europa asociados a lo que Chomsky, mirando a las políticas de Thatcher, denomina "técnica estándar de privatización" compartida por todas esas políticas: infrafinanciar el servicio público que se desea privatizar para conseguir que deje de funcionar bien y tener así la justificación para su privatización; una vez logrado el objetivo, el servicio privatizado funciona peor (Chomsky, 2011). En el caso de la educación como servicio público, la entronización de una "nueva escuela capitalista" gestionada con principios neoliberales que, como evidencian Laval, Vergne, Clèment y Dreux (2012, p. 260), no es "eficaz", que lleva a 
una regresión real de los aprendizajes, y a un incremento agudizado de las desigualdades sociales.

\section{Referencias}

Apple, M. W. (2017). What is Present and Absent in Critical Analyses of Neoliberalism in Education, Peabody Journal of Education, 92, 148-153. Doi: 10.1080/0161956X.2016.1265344.

Astudillo-Ruiz, J. (2004). La trampa partidista de la UGT: de la relación solidaria con el PSOE a la unidad de acción sindical con CCOO. Revista Española de Ciencia Política, 11, 73-101.

Ball, S. J. (1993). What is policy? Texts, trajectories and toolboxes. Discourse, 13(2), 10-17.

Ball, S. J. (2007). Education plc: understanding private sector participation in public sector education. London: Routledge.

Ball, S. J., y Junemann, C. (2012). Networks, New Governance and Education. London: Routledge.

Ball, S. J. y Youdell, B. (2008). La privatización encubierta de la educación pública. Instituto de Educación de Londres. Universidad de Londres.

Bonal, X., Rambla, X., Calderón, E., \& Pros, N. (2005). La descentralización educativa en España. Barcelona: Fundació Carles Pi i Sunyer.

Bourdieu, P. (1998). L'essence du néolibéralisme. Le Monde diplomatique, Mars, 3. https://www.monde-diplomatique.fr/1998/03/BOURDIEU/3609

Bowe, R., Ball, S. J., \& Gold, a. (1992). Reforming Education and Changing Schools: Case Studies in Policy Sociology. London: Routledge.

Cameron, D. (2015). Tory ideas can shape the next decades for all Britons. The Telegraph. https://www.telegraph.co.uk/news/politics/conservative/11804367/davidcameron-tory-ideas-can-secure-britain.html

Cerny, P. (1997). Paradoxes of the competition state: The dynamics of political globalization. Government and Opposition, 32(2), 251-274.

Chomsky, N. (2011). The State-Corporate Complex: A Threat to Freedom and Survival. Text of lecture given at The University of Toronto, April 7.

https://chomsky.info/20110407-2/

Chomsky, N. (2017). Optimism over Despair. London: Penguin Books.

Conservative Party (2010). Invitation to join the government of Britain: The Conservative Manifesto 2010. https://uk. practicallaw.thomsonreuters.com/2-5020351 ?transitionType=Default\&contextData=(sc.Default)

Courtney, S. J. (2016) Post-panopticism and school inspection in England. British Journal of Sociology of Education, 37 (4), 623-642.

Doi:10.1080/01425692.2014.965806 
Cowen, R. (2005). El sistema educativo inglés. En J. Prats, y F. Raventós (Dir.), Los sistemas educativos europeos: ¿crisis o transformación? (pp. 66-87). Barcelona: Fundación La Caixa.

Chitty, C. (2009). Education policy in Britain. New York: Palgrave Macmillan.

DfE (2019). Academies schools' sector in England. London: House of Commons.

Dogan, M. y Pelassy, D. (1990). How to Compare Nations. Strategies in Comparative Politics. London: Chatham House Publishers, Inc, second edition.

Espiet-Kilty, R. (2015). The legacy of Thatcherism in question: An introduction. Observatoire de la société britannique, 17, 11-31. Recuperado de https://journals.openedition.org/osb/1757\#quotation

de Puelles, M. (2005). La influencia de la Nueva Derecha inglesa en la política educativa española (1996-2004). Historia de la Educación, 24(29), 229-253. http://hdl.handle.net/10366/79585

de Puelles, M. (2016). Reflexiones sobre cuarenta años de educación en España o la irresistible seducción de las leyes. Historia y Memoria de la Educación, 3, 15-44. doi:10.5944/hme.3.2016.14760

Fairclough, N. (1992). Intertextuality in critical discourse analysis. Linguistics in Education, 4 (2-3), 269-293. Doi:10.1016/0898-5898(92)90004-G

Fitz, J., y Gorard, S. C. (2002). School Admissions after the School Standards and Framework Act: Bringing the LEAs back in? Oxford Review of Education, 28(2-3), 373-393. Doi:10.1080/03054980220143487

Friedman, M. (1955). The Role of Government in Education, en Robert A. Solo (ed), Economics and the Public Interest. Trustees of Rutgers College. https://la.utexas.edu/users/hcleaver/330T/350kPEEFriedmanRoleOfGovttable.pdf

Friedman, M. (September 23, 1973). Selling schooling like groceries. The New York Times. https://www.nytimes.com/1973/09/23/archives/the-voucher-idea-sellingschooling-like-groceries-vouchers-a-sort.html

Friedman, M. (December 5, 2005). The Promise of Vouchers. The Wall Street Journal. https://www.wsj.com/articles/SB113374845791113764

Giddens, A. (1999). La tercera vía. Archipiélago: Cuadernos de crítica de la cultura, (37), 129-130.

Hilton, A. (2019). Academies and free schools in England: A history and philosophy of the Gove Act. London: Routledge

Hood, C. (1991). A Public Management for all Seasons? Public Administration (69), Spring, 3-19. Doi:10.1111/j.1467-9299.1991.tb00779.x

Klein, N. (2007). La doctrina del "shock". El auge del capitalismo del desastre. Barcelona: Paidós.

Lawton, D. (1994). The Tory Mind on Education 1979-94. London: The Falmer Press.

Lavezzolo, S. y Orrios, L. (2008). El liderazgo de Blair y Aznar hacia el centro político. Revista de Estudios Políticos, 142, 69-103. 
Llanos, A. (2019). Fundamentación versus publificación de la escuela concertada hacia una actualización de las razones de los conciertos educativos. Revista General de Derecho Canónico y Eclesiástico del Estado, 50, 1-31.

Male, T. (2017). Multi-academy trusts (MATs): A background briefing paper. London Center for Leadership in Learning.

Molina-Pérez, A., \& Luengo, J. (2020). Reconstrucciones "Resilientes" de la Identidad Profesional del Profesorado: Endoprivatización y Cultura Performativa en Andalucía (España). REICE. Revista Iberoamericana sobre Calidad, Eficacia y Cambio en Educación, 18(2), 57-75. Doi:10.15366/reice2020.18.2.003

Morin, E. (2016). Enseñar a vivir. Manifiesto para cambiar la educación. Paidós.

Morlino, L. (2018). Comparison. A Methodological Introduction for the Social Sciences. Barbara Budrich Publishers.

National Audit Office (NAO) (2018). Converting maintained schools to academies. London: House of Commons.

Nussbaum, M. C. (2005). El cultivo de la humanidad: una defensa clásica de la reforma en la educación liberal. Barcelona: Paidós.

Ong, A. (2007). Neoliberalism as a Mobile Technology. Transactions of the Institute of British Geographers, 32(1), new series, 3-8. Recuperado de http://www.jstor.org/stable/4639996

Partido Popular (2011). Lo que España necesita: Confianza, Empleo, Reformas, Educación. Más sociedad, mejor gobierno. https://www.pp.es/actualidadnoticia/programa-electoral-pp

Perryman, J., Maguire, M., Braun, A. \& Ball, S. (2018). Surveillance, Governmentality and moving the goalposts: The influence of Ofsted on the work of schools in a post-panoptic era. British Journal of Educational Studies, 66(2), 145-163, DOI:10.1080/00071005.2017.1372560

Pettit, P. (2007). Examen a Zapatero. Madrid: Temas de Hoy.

Prieto, M., y Villamor, P. (2013). Libertad de elección, competencia y calidad: Las políticas educativas de la Comunidad de Madrid. Profesorado. Revista de Currículum y Formación del Profesorado, 16(3), 127-144.

Pulido-Montes, C. (2020). La privatización" de" y" en" la educación pública: un estudio comparado de los casos de Inglaterra y España [Tesis doctoral]. Universidad de Valencia, Valencia.

Rodríguez-Prieto, R. (2012). De la socialdemocracia al socialiberalismo. La socialdemocracia en la encrucijada: declive, renuncias y alternativas. AFD, XXVIII, 293-322.

Stedman, J., D. (2012). Masters of the Universe. Hayek, Friedman, and the Birth of Neoliberal Politics. Princeton, NJ: Princeton University Press.

Torres, J. (2014). Mercado y escuela. Cuadernos de Pedagogía, 445, 58-61.

Torres, J. (2018). Políticas educativas y construcción de personalidades neoliberales y neocolonialistas. Madrid: Morata. 
Tiana, A. (2018). El desarrollo conflictivo del artículo 27 en años decisivos (1982-2008). En M. Puelles- Benítez, \& M. Menor-Currás (Eds.), El artículo 27 de la Constitución: Cuaderno de quejas (pp. 99-120). Madrid: Morata.

Verger, A., Fontdevila, C. y Zancajo, A. (2016). The Privatization of Education. A political Economy of Global Education Reform. Columbia: Teachers College Press.

Verger, A., Prieto, M., Pagès, M., y Villamor, P. (2018). Common standards, different stakes: A comparative and multi-scalar analysis of accountability reforms in the Spanish education context. European Educational Research Journal, 19(2), 142164. Doi:10.1177/1474904118785556

Viñao, A. (2012). El desmantelamiento del derecho a la educación: discursos y estrategias neoconservadoras. Áreas. Revista Internacional de Ciencias Sociales, 31, 97-107.

Viñao, A. (2016). El modelo neoconservador de gobernanza escolar: principios, estrategias y consecuencias en España. En Collet, J. y Tort, A. (Coords.). La gobernanza escolar democrática. Más allá de los modelos neoliberal y neoconservador (pp-41-64). Madrid: Morata.

Weller, W. (2017). Understanding the Operation Called Comparison. Educação \& Realidade, 42(3), 921-937. Doi:10.1590/2175-62366510 